\title{
Wireless Sensor Networks Optimization with Localization-Based Clustering using Game Theory Algorithm
}

\author{
Nina Hendrarini ${ }^{1}$, Muhamad Asvial $^{1}$, Riri Fitri Sari ${ }^{1 *}$ \\ ${ }^{1}$ Department of Electrical Engineering, Faculty of Engineering, Universitas Indonesia, Kampus UI Depok, Depok \\ 16424, Indonesia
}

\begin{abstract}
Wireless sensor networks, as remote monitoring system support or Internet of Things subsystems, require reliability and stability, which greatly influence sensor life spans. Optimization efforts were a breakthrough to obtain these requirements. The optimization carried out in this study is related to the clustering-based localization process. The optimization algorithm used was game theory. Simultaneously, clustering information in an energy availability based sensor node configuration helped the sensor node's tracking process. Optimization in the localization process determined the coalition of anchor nodes, where the selection of nodes as coalition members was conducted through geometric approaches with game theory. This proposed concept was validated using a simulator built on the MATLAB platform. Root Mean Square Error (RMSE) was chosen as a measurement to show accuracy. The simulation results indicated that the number of dead nodes could be delayed by about 1,000 rounds if there are improvements in clustering localization using game theory. The experimental results showed that network performance increased after this cluster-based localization process, which indicated an increase in the number of data packets sent and lifetime of the sensor node. The simulation results for the data delivery test showed a $20 \%$ increase in data packets sent.
\end{abstract}

Keywords: Clustering; Game theory; Localization; RMSE; Wireless sensor networks

\section{Introduction}

A distinctive feature of a wireless sensor network (WSN) is that it consists of many sensors with limited energy availability and is often used over long distances. The sensor nodes have sensing, processing, and communication components in addition to storage capabilities. These sensors work to sense some physical phenomenon, and then the information gathered is processed to get relevant results.

Clustering is the initial network management before the localization process. This assists in decreasing the complexity of managing a large-scale sensor network. This mechanism supplies initial information about the subsequent localization process. Generally, cluster members and cluster heads are based on distance or Received Signal Strength Indicator (RSSI) conversion. The simple mechanism of creating a cluster is achieved by calculating the shortest distance between nodes or from one node to a neighboring node. Then, the maximum eigenvalue radius is calculated to construct a map of the relative locations of the nodes. Clustering can also be determined based on residual 
energy or energy availability. The mechanism for this is the exchange of information between a node and its neighboring nodes. The problem is that determining the distance using the RSSI to distance conversion mechanism still allows errors to occur.

In this research, the objective was to find a suitable localization algorithm design for a WSN that would support the wireless sensors by working stably. In addition, this condition can indirectly reduce energy loss and transmission errors. Ng et al. (2019) determined the factor that reduces the reliability of sensor nodes, which affects the robustness of a network. It also influences the data transmission system of the sensor nodes. They proposed a reliable transport layer protocol that uses a combination of redundant packets and probabilistic transmission to ensure reliable transmission of data to keep the network efficient. Paul and Sato (2017) noted that localization is the provision of physical coordinates for all sensor nodes. Those prior research contributions provided insight for this study through awareness of the various factors and parameters that affect the performance of WSNs by either clustering or localization mechanisms.

Kadi and Alkhayat (2015) discuss many other factors that contribute to late errors in the localization process, such as network density, uncertainty about anchor locations, and density. The placement of anchor nodes in a coalition is an essential prerequisite for accuracy in the sensor nodes' localization process. Thimmaiah and Mahadevan (2017) clarified that this accuracy is necessary in military, mining, and industrial applications. For example, knowing the exact position of an intruder that is considered the target node is crucial in military operations. They also described a relationship between the transmission failure probability (Marcum's function value) and the standard deviation of the localization error. The localization error also impacts geographic routing. A comprehensive perspective on the effect of localization errors is given by taking into account the quality of service parameters. Sachidananda et al. (2010) determined that a low probability of transmission failure improves the quality of the information in terms of reliability and timeliness. The novelty in this study is considering the potential fit in the initial view based on the geometry factor of the previous initial experiment. In localization, the game theory algorithm is used to refine the anchor nodes in the coalition. Determining the number of anchor nodes and their placement greatly affects the localization performance. In a network with many sensors and extensive scalability, choosing anchor nodes has its own strategy. That strategy can be considered the game's rules in game theory, while the sensor node can be considered a player. Earnings in the game can be considered to be the probability of a node becoming an anchor node.

The problem's background, according to Hadzic et al. (2013), is that many people assume good localization results will occur in a dense sensor network with many anchor nodes. However, the use of enormous power for transmitting and processing all information does not guarantee good localization performance. Hadzic et al. (2013) found that not all anchor nodes provide reliable measurements, but the geometry of a selected reference node has been shown to have a significant impact on localization accuracy. They overcame this problem by assuming that the target node has several anchor nodes nearby, taking a different view. They then used the Cramer-Rao lower bound (CRLB) to capture information about node geometry and ranging quality, such as the quality of distance estimates obtained from noisy measurements received. They model the localization process as a cooperative game and formulate the appropriate utility function, defining node selection optimization as a process that maximizes accuracy. To find anchor nodes they use utility functions. Our study used several strategies to conduct a series of performance evaluations of the localization with various anchor nodes, placing the anchor node near the target node. The first strategy was determining the coalition formed with a minimum of 
three nodes. If the number of anchor node coalitions was less than three, the quality of the coalition was zero. The utility function in our study was obtained by calculating the amount of energy used for communication and computing, along with the anchor node suitability factor, which was first calculated using the Nash equilibrium approach. The second strategy was to use the utility function as a weighted factor to obtain a target node's signal range value that was closer to the actual condition. By knowing the radius of the target node, it was possible to estimate the area where the prospective anchor node was located. The third strategy was the geometric method of placing anchor nodes around the target node. Three conditions affect the magnitude of error during localization: whether the topology is in the form of a right triangle, an acute triangle, or an obtuse triangle. In this case, the anchor node topology is not collinear. The concept of a non-collinear anchor node topology was also raised by Sun et al. (2018), who proposed an error control mechanism based on anchor geometric features to determine the nodes that participate in the localization and to estimate the localization confidence.

The remainder of this paper is organized as follows: Section 2 is a brief overview of clustering techniques, localization, game theory, and methodologies used in the localization optimization process. Section 3 presents the process of experimenting and testing clusterbased localization methods with game theory algorithms. Finally, Section 4 summarizes the paper and explains future work.

\section{Methods}

The clustering process can be helpful because it provides initial location information that is more organized and patterned. This condition follows the optimization objectives related to energy savings. The next step was processing this initial information with the obstructions indicated by the signal strength received using the method of localization. The localization quality is indicated by the cooperation between anchor nodes when tracking the position of target nodes or unknown nodes. Factors that affect the quality of the coalition between these anchor nodes are the geometric locations of the anchor nodes, their distance, and signal strength. The novelty offered by this research is the combination of the clustering and localization processes to manage networks using a game theory algorithm.

\subsection{Clustering Protocol}

Sarobin and Thomas (2016) delivered Low Energy Adaptive Clustering Hierarchy (LEACH) algorithm improvements based on energy efficiency. In their research, the cluster head selection process took into consideration the residual energy and the node's distance to the sink. At the same time, the non-cluster head nodes had to be within the cluster head's transmission range in the cluster formation process.

Reddy and Raja (2017) implemented the distributed energy-efficient clustering (DEEC) protocol to improve the performance of heterogeneous sensor networks. There are data from sensor nodes in the form of residual energy nodes at each epoch, which allows for the calculation of the probability of the node becoming a cluster head. The probability for the sensor node to be a cluster head is calculated by considering the node's residual energy every rotation compared with the network's average energy. With the average energy of the node $\left(E_{a v g}\right.$ ) considered, the energy consumption calculation for further distances will use more energy. Cluster heads are chosen based on probabilities calculated with the ratio of each node's residual energy to the average energy of the $E_{\text {avg }}$ network. The total initial energy of a three-level heterogeneous network is calculated using Equations 1 and 2.

$$
\begin{gathered}
E_{\text {total }}=E_{\text {normal }}+E_{\text {advance }}+E_{\text {super }} \\
E_{\text {total }}=N(1-m) E_{o}+N_{m}\left(1-m_{o}\right)(1+a) E_{o}+N \cdot m \cdot m_{o}(1+b) E_{o}
\end{gathered}
$$


where the energy allocation pattern of the total energy $E_{\text {total }}$ consists of the energy for a normal node $E_{\text {normal }}$, energy for an advanced node $E_{\text {advance, }}$ and energy for a supernode $E_{\text {super. }}$. The percentage of an advanced node is $m$, the percentage of the supernode is $m_{0}$, the percentage energy of the advance node is a, and the percentage energy of the supernode is b. The initial energy is $E_{o}$. The initial probability $\mathrm{P}_{\text {opt }}$ is a next cluster head probability $\mathrm{Pi}$ node's reference. Equation 3 shows the probability of a node being a cluster head:

$$
\mathrm{Pi}=\mathrm{P}_{\text {opt }}\left(1-\frac{\mathrm{E}_{\text {avg }}-\mathrm{E}(\mathrm{i})}{\mathrm{E}_{\text {avg }}}\right)
$$

The average energy of the network can be found using Equation 4, and the probability average $\mathrm{P}_{\text {avg }}$ is shown in Equation 5.

$$
\begin{gathered}
\mathrm{E}_{\mathrm{avg}}=\frac{1}{\mathrm{~N}} \sum_{1}^{\mathrm{N}} \mathrm{Ei} \\
\mathrm{P}_{\mathrm{avg}}=\frac{1}{\mathrm{~N}} \sum_{1}^{\mathrm{N}} \mathrm{Ei}\left(1-\frac{\mathrm{r}}{\mathrm{R}}\right)
\end{gathered}
$$

The localization involves several factors, such as techniques for estimating the position of sensor nodes distributed in the field of observation where some have position information. Localization also determines or defines the node's physical position within some coordinate system or symbolic location. Terminology of position should be agreed upon before there can be an understanding of localization. Global position refers to the position in the global frame of reference with the global positioning system (GPS; longitude and latitude) or the Universal Transverse Mercator (UTM; zone and latitude). Next is the relative position, which is based on an arbitrary coordinate system, and the distance between sensor nodes, which is not related to global coordinates.

The performance of this localization mechanism is shown by the level of accuracy of the position tracking results, which depends on the anchor node performance. Trilateration is a technique used to trace RSSI-based nodes by using a minimum of three known reference node positions. Generally, anchor nodes are equipped with GPS, which means that the cost is relatively high. The first stage of the trilateration process involves estimating unknown target nodes using the RSSI computational approach with the effect of path loss and GPS positioning of the anchor node. The subsequent stage involves the tracking process using a coalition of anchor nodes to obtain the coordinates of the target nodes, which are the intersections of the three axes of the triangle according to the Euclidian equation. Figure 1 depicts the trilateration technique in two dimensions and the multilateration technique in three dimensions.

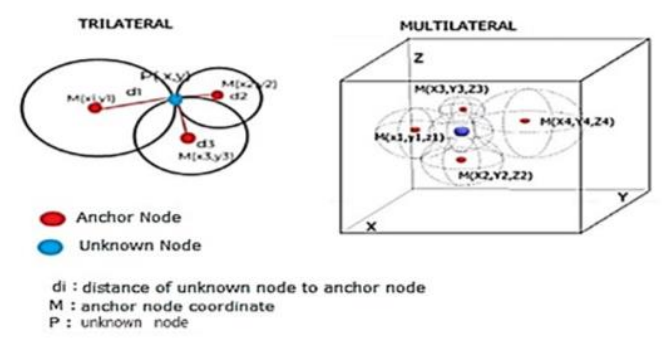

Figure 1 Two-dimensional trilateration and three-dimensional multilateration

Shah et al. (2018) tracked the position of a target using RSSI in three dimensions to determine the exact distance. The localization process was a multilateration approach that used a minimum of four anchor nodes. The multilateration math equation is shown in Equation 6.

$$
\mathrm{di}^{2}=(\mathrm{x}-\mathrm{xi})^{2}+(\mathrm{y}-\mathrm{yi})^{2}+\left(\mathrm{z}-\mathrm{z}_{1}\right)^{2}
$$


where the coordinates of the anchor nodes are $\mathrm{M}_{1}\left(\mathrm{x}_{1}, \mathrm{y}_{1}, \mathrm{z}_{1}\right), \mathrm{M}_{2}\left(\mathrm{x}_{2}, \mathrm{y}_{2}, \mathrm{z}_{2}\right), \mathrm{M}_{3}\left(\mathrm{x}_{3}, \mathrm{y}_{3}, \mathrm{z}_{3}\right)$, and $\mathrm{M}_{4}\left(\mathrm{x}_{4}, \mathrm{y}_{4}, \mathrm{z}_{4}\right)$. The unknown node or target node coordinate is $\mathrm{P}(\mathrm{x}, \mathrm{y}, \mathrm{z})$. In the localization, root mean square error (RMSE) is a measure of accuracy because it shows the forecast error for a specific data set and not between data sets. RMSE can be calculated using Equation 7 .

$$
\text { RMSE }=\sqrt{\frac{\sum_{1}^{N}(x i-x p)^{2}}{N}}
$$

which shows that the number of nodes is 1 to $\mathrm{N}, \mathrm{x}_{\mathrm{i}}$ is the estimated sensor node coordinate, and $\mathrm{x}_{\mathrm{p}}$ is the node reference coordinate. Optimization carried out in this localization is the arrangement of anchor nodes in placement or in other arrangements. The emphasis with this anchor node is still related to energy use.

The localization process is divided into two phases. First is the ranging or estimating phase. As shown in Figure 2, this phase illustrates the process of estimating the distance from the signal received by a target node. The second phase uses the estimated distance to get the position of the node, depending on certain equations or optimized algorithms. The accuracy value indicates how close the detected node's position was in comparison to the estimation. The importance of accuracy if placements are slightly shifted, such as by a few centimeters to the left or right in localization, is related to the WSN's implementation and purpose. WSNs is attractive if implemented specifically in hazardous environments and in large numbers. If the sensor implementation is only for monitoring acidity on the walls of a building, it may not be difficult to place, and the localization accuracy is not particularly significant. The situation is different if a WSN is implemented in the military. The development of WSNs in the military is rapidly growing because the forerunner of WSNs was developed by the military, and currently, this type of network is used for various purposes in the military field.

Ali et al. (2020) divided the military uses of WSNs into three categories: combat monitoring, battle surveillance, and intruder detection. One example implementation is laying land mines, which includes the challenges of detecting the positions of land mines so that they are in an accurate position and in accordance with the military operation plan. On today's battlefields, the use of sensor networks, along with other sophisticated devices, is essential. The use of a WSN can reduce uncertainty or inaccuracy in the positions of troops or equipment, which makes it easier to set strategies to avoid disasters or reduce risks. Thus, a WSN's performance is critical in military implementations, particularly localization accuracy because a fatal risk can occur due to an inaccurate position of a node.

\subsection{Game Theory}

Game theory has the advantage of being a multi-agent decision problem. But a challenge exists in choosing the appropriate utility function in the analytical model. Random data are initially processed with a selection mechanism according to the desired strategy, whether searching for the maximum-minimum value, looking for fluctuating values, finding the saddle point, or other provision. Then, the natural tendency of the game is reviewed. An appropriate strategy is sought based on various game theory characteristics, including cooperative or non-cooperative behavior, zero-sum, or other variations.

Ejidokun et al. (2019) put forward that the solution for a real-world problem involves first translating it into a mathematical approach or mathematical model. The system model is the result of a comprehensive systems analysis of engineering expertise. Explicit analysis of the objectives to be achieved, significant parameters, symptoms, and correlations are the main elements required to find solutions. Game theory is one strategy for translating problems in actual conditions into mathematical models. In past studies, Attiah et al. (2017) 
selected cluster heads using clustering algorithms for efficient energy use, thereby extending sensor node life. Sensor nodes in a network that consume different energies need to use some rational energy use scheme to regulate network connectivity and function. Pitchai et al. (2019) proposed a game theory based energy-efficient routing algorithm (GERA). They adopted game theory and set a utility function based on the benefit and energy consumption of the node in the clustering mechanism. They also used the Nash equilibrium to determine the relationship between utility and play strategy.

\subsection{Clustering-Based Localization}

In initial conditions, the distribution of sensor nodes will be managed in a cluster where available energy is taken into consideration using a distributed clustering protocol. Initial information in the form of cluster placement will be the initial data set in localization. The next stage is determining the physical location of the sensor node by considering the signal strength received. The selection and placement of anchor nodes in the coalition affect the accuracy of determining the target sensor node's position. In the present study, a game theory algorithm was used for efficiency in determining this coalition. In addition to modeling localization with game theory, we looked for correlation formulations between utility functions. The utility function was defined as a geometric representation of nodes, distance, and position ranges. The computational complexity was reduced by using a random search method that takes advantage of the spatial correlation among coalition members.

\subsection{Anchor Node Coalition with Game Theory}

We used game theory for coalition optimization, where the utility function was a parameter optimized by game theory. The utility function is an exchange between energy and available cost-the greater the utility, the better the performance. Coalitions have utility functions that describe the correlation between players, strategies, and outcomes. Initially, the network formed a single coalition. The subsequent process involved joining two single coalitions under certain conditions, which allowed the nodes to communicate with each other and reach an agreement to be combined. Coalition formation with the game theory model was directed at the utility function convex optimization approach. Finding equations that allow the determination of utility can be achieved in scoring mapping, as shown in Table 1.

Table 1 Coalition payoff matrix

\begin{tabular}{cccc}
\hline & & \multicolumn{3}{c}{ Node 1 } \\
\cline { 3 - 4 } & & $(1-\Psi)$ & $\Psi$ \\
\hline$N$ & $\varphi(S)$ & $(1,1)$ & $(0,0)$ \\
$\tilde{0}$ & $\eta(S)$ & $(0,0)$ & $(1,1)$ \\
\hline
\end{tabular}

The general pattern of the equilibrium indicates the entity relationship:

$$
(1-c) \alpha+c(\beta)
$$

The possibilities for implementing the strategy are denoted by c; if that is not achieved, then the probability is (1-c), considering that the values of $\alpha$ and $\beta$ are the payoff. A relationship is determined by using the general form of the relationship between these variables when linked to a map. The meaning of the map is that the quality of the coalition depends on mismatches between coalition members. Based on this relationship, the Nash equilibrium can be found. A relationship is a mixed strategy involving the suitability of conditions, the proximity of the node positions, and the coalition's quality. This correlation is expressed by the mathematical model shown in Equation 9. 


$$
(1-\Psi) \varphi(S)+\Psi \cdot \eta(S)
$$

If $S$ is the coalition symbol, which is a subset of the number of $\mathrm{N}$ nodes, then the coalition value can be determined. This difference is denoted by $\Psi$, which has a value between zero and one. This difference will determine the likelihood of a node joining the coalition. Here, the boundary value shows the relationship between the parameters.

$$
\text { then } \quad v(S)=\left\{\begin{array} { c } 
{ 0 } \\
{ \varphi } \\
{ ( 1 - \psi ) \varphi ( S ) + \psi \cdot \eta ( S ) }
\end{array} \quad \left\{\begin{array}{l}
|S|=1 \\
|S|=3 \\
|S| \geq 4
\end{array}\right.\right.
$$

The utility function $v(S)$ has a value of zero when in a singleton coalition, which means there is no tracking process of unknown nodes. If the utility function has a value of $\varphi$ when there are three anchor nodes, a tracking process has occurred even at minimum conditions. The symbol $\varphi$ indicates the relationship between the three anchor nodes in trilateration. The symbol $\eta(S)$ indicates the anchor node and unknown node closeness, and the symbol $\varphi(\mathrm{S})$ indicates the anchor nodes' coalition quality. By looking at Figure 2, the proximity of anchor nodes and coalition quality can be estimated. The actual distance from the trilateration first iteration results is $d_{i}{ }^{\prime}$, while $d_{i}$ is the unknown node's estimated distance. Figure 2 shows the definition of the radius of the signal range of the unknown node, denoted by $D$, while $d_{i j}$ is the distance between the unknown node and each anchor node in the unknown node radius range.

In this study, sensor node selection becomes a coalition of anchor nodes by achieving the best response condition through the Nash equilibrium. The first strategy in selecting this anchor node coalition is to determine if the coalition consists of a minimum of three anchor nodes. The second strategy is whether anchor nodes are located within a signal range from the target node. The third strategy is whether the geometric correlation between the three anchor nodes meets the geometric rules indicated by the distance between the anchor nodes as a side triangle, an equilateral triangle, an acute triangle, or a right triangle.

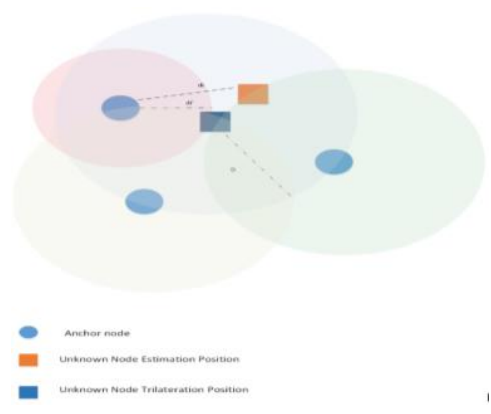

Figure 2 Distance configuration

The coalition quality value of anchor nodes can be calculated using Equation 12 .

$$
\varphi(\mathrm{S})=1-\frac{1}{\mathrm{~N}} \sum_{1}^{\mathrm{N}} \frac{\mathrm{di} \prime}{\mathrm{di}}
$$

The proximity value of the anchor node and the unknown node is shown in Equation 13.

$$
\eta(S)=\frac{1}{M} \sum_{i, j}^{M} \frac{\text { dij }}{D}
$$

where $\mathrm{v}(\mathrm{S})$ as a weighting factor affects the initial radius of the unknown signal. Node D becomes $d$ with the value

$$
\mathrm{d}=\mathrm{v}(\mathrm{S}) \times \mathrm{D}
$$


The anchor node location for prospective coalition members can be found by determining the target node radius.

Figure 3 shows the process of selecting anchor nodes to be coalition members. Based on the experiment, it is estimated that the anchor node located at the limit of signal coverage and with radius D will produce relatively good accuracy of position tracking. Typical localization techniques are usually performed assuming a line-of-sight (LOS) environment. However, LOS signals are typically subject to some reflection and diffraction. The technique used in the present study looked for a solution with a distributed localization algorithm. Localization was carried out with the initial information from clustering in a distributed manner and modeled in the geometric relationship of the anchor node position with the measurement of signal range. There is a cooperative exchange of information between anchor nodes in a distributed localization algorithm. This assumption makes the value of $\mathrm{D}$ a relative range value. Knowing the transmission energy can es timate the value of the range D with the LOS situation assumption. A correction factor will give an actual picture of the existence of signal strength with external influences. Hence, the anchor node located in the circle with a radius of $\mathrm{d}$ was selected.

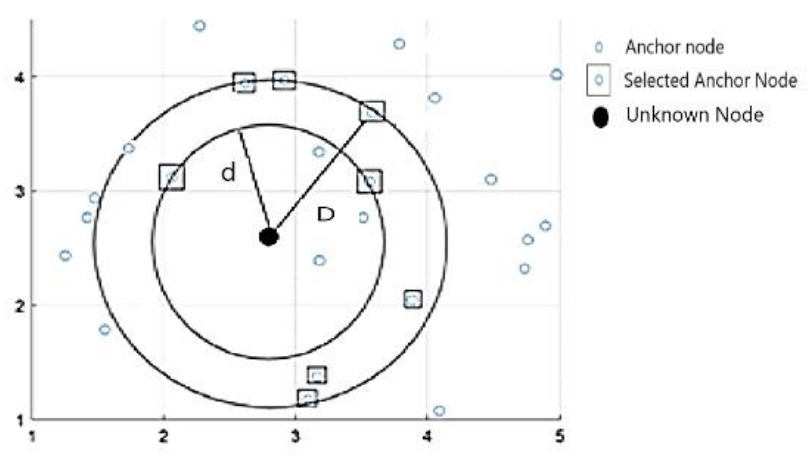

Figure 3 Selection of anchor nodes

The selection of anchor nodes for a coalition must not be collinear. Although it is located within the signal coverage radius, it is not selected as a coalition member. The coalition forming concept using anchor nodes accompanied by GPS with reference nodes from the trilateration results begins with determining the radius of the signal range from the target node where the coordinates of its position will be sought. Determining the distance between the target node and the anchor node requires a communication mechanism; hence, this information about the distance is learned through the computation process. Based on this information, anchor nodes will be processed in collaboration to track the position, and anchor nodes that are located in the target node signal radius range have the potential to become members of the coalition. Then the anchor node will look for neighboring nodes that will form a coalition, with a minimum of three anchor nodes required to perform trilateration. In the initial conditions, the first element to become a reference is the signal range from the unknown node. Then the value of $d$, which is the signal range value after adjustment, will be determined.

\section{Results and Discussion}

This section discusses the testing and validation process, where the material being tested was related to the clustering and localization process, with several parameters for MATLAB simulation. 


\subsection{Coalition Formation in Localization}

A soft experiment was carried out on several sensor node trilaterations using a MATLAB-based simulator before looking for the best conditions for placing anchor nodes in a coalition. This was done because finding the most appropriate anchor node coalition is complex enough.

Table 2 Localization parameter settings

\begin{tabular}{lc}
\hline \multicolumn{1}{c}{ Parameter } & Value \\
\hline Network area & $1,000 \mathrm{~m} \times 1,000 \mathrm{~m}$ \\
Unknown nodes & 10 \\
Anchor nodes & 3 \\
Propagation model & Two-ray ground \\
\hline
\end{tabular}

Figure 4 shows the localization results with a specific anchor node configuration. The relationship between coalition anchor nodes and the position of anchor nodes located on a circle with an unknown node signal coverage radius is exhibited. It is assumed that the center of the circle is a red dot. This point is located nearby the sensor node whose coordinates are unknown. This means that there are factors influencing the determination of coalition nodes that will be found using game theory, assuming ideal conditions where anchor nodes are located within the maximum signal range of the unknown node.

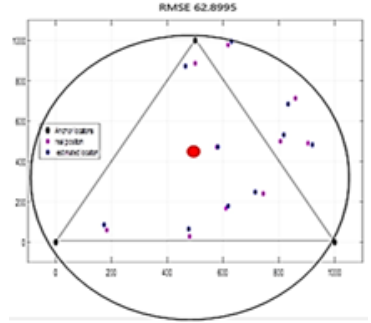

(a)

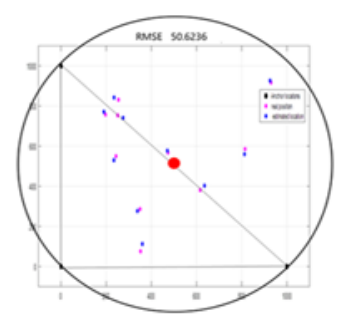

(b)

Figure 4 (a) Anchor node placement, and (b) signal coverage radius of target node

Figure 4a indicates the anchor nodes' circular locations, with coalition formation in equilateral triangles. The RMSE of this configuration is $62.8995 \mathrm{~m}$, while the coalition formation in Figure $4 \mathrm{~b}$ is an isosceles right triangle with an RMSE of $50.6236 \mathrm{~m}$. If an analysis of the simulation results is carried out, it will turn out that there is a correlation between the geometric appearance of the placement of anchor nodes in the coalition with the accuracy of the nodes' locations.

\subsection{Localization Clustering with Anchor Node Placement}

A distributed clustering study was conducted for re-experiments related to the clustering mechanism, the selection of cluster heads, and cluster formation. The localization process was carried out based on clustering, starting with the anchor node placements. In the localization process, it was necessary to adjust the parameters shown in Table 3.

Table 3 Localization clustering-based parameter settings

\begin{tabular}{|c|c|}
\hline Parameter & Value \\
\hline Area & $1,000 \mathrm{~m} \times 1,000 \mathrm{~m}$ \\
\hline Number of nodes & 50 \\
\hline $\mathrm{E}_{o}$ (initial energy) & $0.5 \mathrm{~J}$ \\
\hline $\mathrm{E}_{\mathrm{da}}$ (aggregation energy) & $5 \mathrm{pJ} / \mathrm{bit} / \mathrm{signal}$ \\
\hline Etx (transmitter energy) & $50 \mathrm{pJ} / \mathrm{bit}$ \\
\hline $\mathrm{E}_{\mathrm{rx}}$ (receiver energy) & $50 \mathrm{pJ} / \mathrm{bit}$ \\
\hline
\end{tabular}




\begin{tabular}{lc}
\hline \multicolumn{1}{c}{ Parameter } & Value \\
\hline a (energy fraction) & 0.03 \\
Efs (free space energy) & $0.02 \mathrm{pJ} / \mathrm{bit} / \mathrm{m}^{2}$ \\
Emp (multi-path energy) & $0.05 \mathrm{pJ} / \mathrm{bit} / \mathrm{m}^{4}$ \\
Packet data length & $3,000 \mathrm{bits}$ \\
Number of anchor nodes & 4 \\
N (Round number) & 5,000 \\
Gaussian constant (free space) & 2 \\
\hline
\end{tabular}

Figure 5 shows that four anchor nodes have been placed in the space. Placing anchor nodes in the corner of the room is a general localization step when assuming the signal covers almost all nodes.

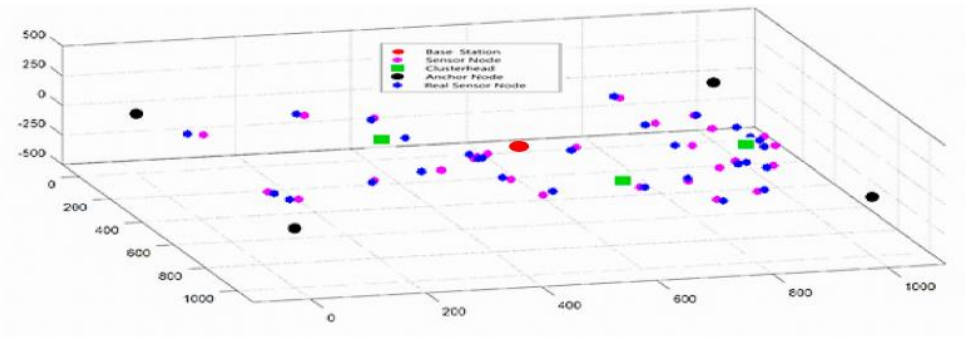

Figure 5 Localization with anchor node placement

Figure 5 shows the multilateration result, where there is a difference between the estimated node position and the actual node. The smaller the difference, the better the accuracy.

\subsection{Localization with Anchor Node Coalition using Game Theory Algorithm}

For the present study, the coalition arrangement in the localization process used game theory, as shown in the following simulation results. The review is visualized in two dimensions so that the position of the anchor node in a coalition is obvious.

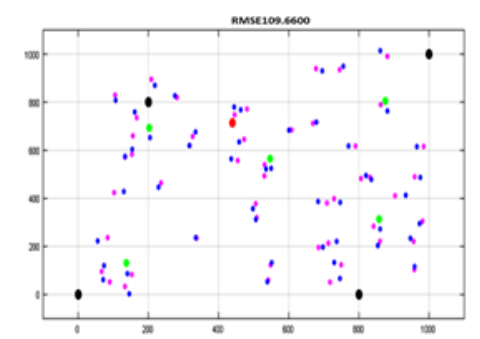

(a)

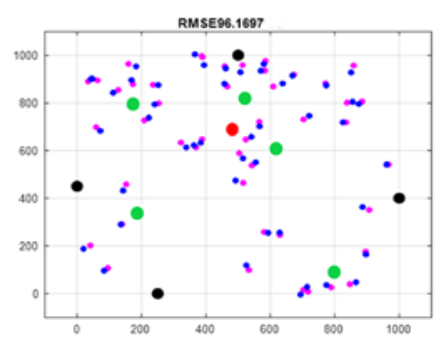

(b)

Figure 6 Clustering and localization with coalition game theory

Figure 6a shows an anchor node coalition that has not been adjusted to game theory, which had an RMSE of $109.66 \mathrm{~m}$. In contrast, Figure 6b shows the anchor node adjusted to game theory, with an RMSE of $96.19 \mathrm{~m}$. The RMSE value resulting from coalition localization using game theory was lower than a random condition, so it tends to be more accurate. The geometric configuration of anchor nodes in the coalition can explain the accuracy of the nodes; if the topology is closer to the square shape, it will provide a better level of accuracy. Figure 6a shows that the anchor node configuration in the coalition tended to form a parallelogram. Meanwhile, in Figure 6b, the configuration tended to be square.

The test scenario of Figure 7 is regarding the data transmission process after the clustering-based localization process was completed. The final result can be viewed from the parameters of the number of data packets sent to the base station and the number of 
still alive nodes at a time.

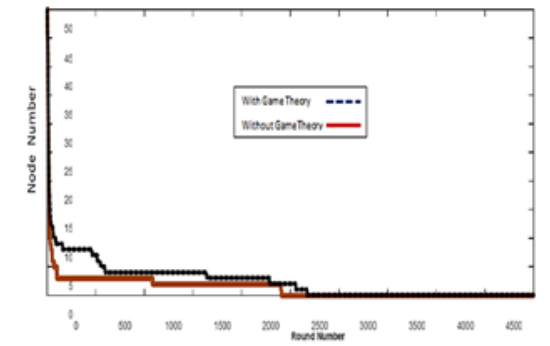

(a)

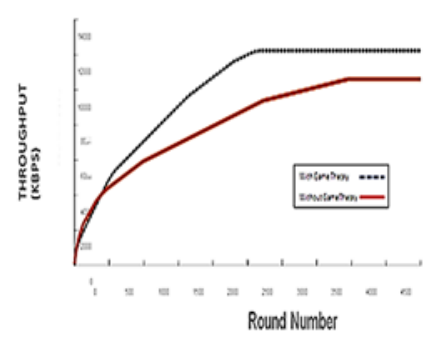

(b)

Figure 7 (a) Live nodes vs. round number; (b) throughput vs. round number

The comparisons made during the experiment were localization using game theory and random algorithms that did not use game theory. Figure $7 \mathrm{a}$ is a graph indicating the number of sensor nodes that were still alive during the review, when the network had been clustering with localization using game theory. The result was a longer node life span of about 1,000 rounds. Figure $7 \mathrm{~b}$ depicts the test scenario with regard to the number of data throughputs; the number of throughputs increased around $2 \times 10^{3} \mathrm{kbps}$ when the localization was adjusted with game theory and without game theory.

\section{Conclusions}

In implementing a distributed clustering protocol application, problems can arise when there is an imbalance in energy consumption due to a non-uniform distribution of nodes or where nodes have different densities. As a solution, it is proposed to set various optimization mechanisms that determine the boundary values associated with the available energy, and the computation is completed using game theory. During the sensor network's localization, problems can also occur with determining the anchor node coalition to carry out a trilateration or multilateration process. The approach is heuristic by combining game theory and geometric methods to obtain boundary anchor nodes for prospective coalition members. The simulation results indicated that the number of dead nodes could be delayed by about 1,000 rounds if there are improvements in clustering localization with game theory. The experimental results also show that network performance tended to increase after this cluster-based localization process, indicating an increase in the number of data packets sent and the longer lifetime of the sensor node. The simulation results validated a $20 \%$ increase in data packets sent.

\section{Acknowledgements}

This work was supported by a doctoral dissertation research grant from the Ministry of Research, Technology, and Higher Education (Kemristekdikti) PDUPT 2019 with contract number 2552854.

\section{References}

Ali, A., Changaz,i S.A., Khan, Y.J., Muhammad Qasim, 2020. Game Theoretical Computation Military Operations: Wireless Sensor Networks based Applications to Reinforce Future Battlefield Command System. In: 2020 IEEE 23 ${ }^{\text {rd }}$ International Multitopic Conference (INMIC)

Attiah, A., Chatterjee, M., Zou, C.C., 2017. A Game-Theoretic Approach for Energy-Efficient Clustering in Wireless sensor networks. In: 2017 IEEE Wireless Communications and Networking Conference (WCNC) 
Ejidokun, O.T., Onibonoje, M.O., Ojo, A.O., 2019. A Mathematical Modeling Approach for Optimal Trade-offs in A Wireless Sensor Network for A Granary Monitoring System, International Journal of Technology, Volume 10(2), pp. 332-338

Hadzic, S., Rodriguez, J., Mumtaz., 2013. Cooperative Game theory and Its Application in Localization Algorithms. IntechOpen. https://doi.org/10.5772/53930

Kadi, M., Alkhayat, I., 2015. The Effect of Location Errors on Location-Based Routing Protocols in Wireless Sensor Networks. Egyptian Informatics Journal, Volume 16(1), pp. 113-119

Ng, J.R., Goh, V.T., Yap, T.T.V., Shuib, M.K., 2019. Adaptive Redundancy-Based Transmission for Wireless Sensor Networks. International Journal of Technology, Volume 10(7), pp. $1355-1364$

Paul, A.K., Sato, T., 2017. Localization in Wireless Sensor Networks: A Survey on Algorithms, Measurement Techniques, Applications, and Challenges. Journal of Sensor and Actuator Networks, Volume 6(4), pp. 1-23

Pitchai, K.M., Paramasivan, B., Bhuvaneswari, B., 2019. Game Theoretical Computation based Energy Efficient Routing for Wireless Sensor Networks. In: $3^{\text {rd }}$ International Conference on Eco-friendly Computing and Communication Systems

Reddy, M.M.P., Raja, S.V., 2017. DEEC Protocol for WSNs. India Advances in Wireless and Mobile Communications, Volume 10(1), pp. 51-63

Shah, S.B.H., Zhe, C., Yin, F., Khan I.U., 2018. 3D Weighted Centroid Algorithm RSSI Ranging Model Strategy for Node Localization in WSN based on Smart Devices. Sustainable Cities and Society, Volume 39, pp. 298-308

Sarobin, M.V.R. Thomas, L.A., 2016. Improved Leach Algorithm for Energy Efficient Clustering of Wireless Sensor Network (WSN). International Journal of Technology, Volume 7(1), pp. 50-60

Sachidananda, V., Khelil, A., Suri, N., 2010. Quality of Information in Wireless Sensor Networks: A Survey. In: $15^{\text {th }}$ International Conference on Information Quality

Sun,Y., Wang, X., Yu, J., Wang,Y., 2018. Heuristic Localization Algorithm with a Novel Error Control Mechanism for Wireless Sensor Networks with Few Anchor Nodes. Journal of Sensors, Volume 2018, https://doi.org/10.1155/2018/5190543

Thimmaiah, S.H., Mahadevan, G., 2017. A Range Based Localization Error Minimization Technique for Wireless Sensor Network. Indonesian Journal of Electrical Engineering and Computer Science, Volume 7(2), pp. 395-403 